\title{
Umbilical cord blood transplants facilitated by the French cord blood banks network. On behalf of the Agency of Biomedicine, Eurocord and the French society of bone marrow transplant and cell therapy (SFGM-TC)
}

\author{
Hanadi Rafii $\mathbb{I}^{1,2} \cdot$ Federico Garnier ${ }^{3} \cdot$ Annalisa Ruggeri $\mathbb{( D}^{1,4} \cdot$ Irina lonescu $^{3} \cdot$ Caroline Ballot $^{5}$. \\ Danièle Bensoussan ${ }^{6} \cdot$ Christian Chabannon $\mathbb{1}^{7} \cdot$ Bernard Dazey $^{8} \cdot$ John De Vos $^{9} \cdot$ Eric Gautier $^{10}$. \\ Christine Giraud ${ }^{11} \cdot$ Jérome Larghero ${ }^{12}$ - Audrey Cras $\mathbb{1}^{12} \cdot$ Valérie Mialou $^{13} \cdot$ Virginie Persoons $^{14}$. \\ Fabienne Pouthier ${ }^{15}$. Jean-Baptiste Thibert ${ }^{16}$. Jean-Hugues Dalle $\mathbb{D}^{17}$. Gerard Michel $\mathbb{D}^{18}$. Chantal Kenzey ${ }^{1,2}$. \\ Fernanda Volt ${ }^{1,2}$ - Vanderson Rocha ${ }^{1,19}$ - Jacques-Olivier Bay ${ }^{20}$ - Marie-Thérèse Rubio ${ }^{21}$ - Catherine Faucher ${ }^{3}$. \\ Evelyne Marry ${ }^{3} \cdot$ Eliane Gluckman ${ }^{1,2}$
}

Received: 10 October 2020 / Revised: 4 March 2021 / Accepted: 13 April 2021 / Published online: 14 May 2021

(c) The Author(s), under exclusive licence to Springer Nature Limited 2021

\begin{abstract}
The public French Cord Blood Banks Network was established in 1999 with the objective of standardizing the practices governing umbilical cord blood (UCB) banking in France. The Network adopted a strategy to optimize its inventory and improve the quality of its banked units based on a quality improvement process using outcome data regularly provided by Eurocord. This study aimed to describe the results, over 10 years, of UCBT facilitated by a national network that used the same criteria of UCB collection and banking and to assess how modifications of banking criteria and unit selection might influence transplant outcomes. Nine hundred and ninety-nine units (593 single-unit and 203 double-unit grafts) were released by the Network to transplant 796 patients with malignant $(83 \%)$ and non-malignant (17\%) diseases. Median cell dose exceeded $3.5 \times 10^{7} \mathrm{TNC} / \mathrm{kg}$ in $86 \%$. There was a trend to select units more recently collected and with higher cell dose. Neutrophil engraftment was $88.2 \%(85.7-90.7)$ and $79.3 \%$ (72.6-86.5) respectively for malignant and non-malignant diseases with a trend to faster recovery with higher cell doses. The respective 3-year transplant-related mortality were $31.1 \%$ (27.5-35.1) and 34.3\% (27.0-43.5). OS was $49 \% \pm 4$ in malignant and $62 \% \pm 4$ in non-malignant disorders. In multivariate analysis, cell dose was the only unit-related factor associated with outcomes. Our results reflect the benefit on clinical outcomes of the strategy adopted by the Network to bank units with higher cell counts.
\end{abstract}

\section{Introduction}

Since the first cord blood transplant [1], improvement in umbilical cord blood (UCB) unit selection resulted in better outcomes after both single- and double-unit cord blood transplants [2-5]. Major limitations remain delayed hematopoietic recovery and high incidence of graft failure, resulting in an increased risk of non-relapse mortality compared to other stem cell sources [6-11]. To date, HLA matching and cell dose are the most important criteria when

Hanadi Rafii

hanadi.rafii-elayoubi@aphp.fr

Extended author information available on the last page of the article selecting UCB units for transplantation [12-17]. Historically, HLA was typed based on HLA-A, -B antigen level, and -DRB1 allele level. Subsequently, it was demonstrated that HLA-C match and high-resolution were important factors for outcomes. Increasing the total nucleated cell (TNC) above the recommended minimum pre-freezing dose of $2.5-3 \times 10^{7} / \mathrm{kg}$ has also been suggested to improve engraftment and survival $[17,18]$. The French Cord Blood Banks Network (in French, Réseau Français de Sang Placentaire, called the Network throughout the article) was established in 1999 under the auspices of the National Agency for Organs and Bone Marrow Transplantation later replaced by the Agency of Biomedicine (ABM), with the aim of standardizing practices governing collection, storage, and release of UCB units across the national cord blood 
banks (CBB) [19]. In France, cord blood banking can only be performed in public banks authorized by competent National Health Authorities to process and store UCB units. For this propose, standard criteria for cord blood banking that resulted in collection and storage of UCB units of similar quality have been established and implemented through regular meetings and discussions organized by $\mathrm{ABM}$ between the CBBs, maternity wards, and transplant physicians. Methods for collection, cryopreservation and thawing, measurement of cell dose, and viability were standardized to fulfill the Foundation for the Accreditation of Cellular Therapy (FACT) - NetCord standards adopted by the Network [20]. The Network recommendations have been modified overtime based on transplant outcome data regularly provided by Eurocord and used as quality improvement indicators that assess the performance of the Network.

This study aims to describe the characteristics and distribution in quality categories of the French UCB units collected and released over a period of 10 years and to analyse the outcomes of transplants performed with these units during the same period, considering the modifications of the banking criteria carried out in the context of the quality improvement process, in terms of standardized collection and processing policies, pre-freezing unit eligibility criteria (collected volume, minimal TNC, and CD34 counts) and HLA typing.

\section{Materials and methods}

In this registry-based retrospective study, we included all consecutive patients who received unrelated single or double UCBT (sCBT or dCBT) in an EBMT center, with units released by the Network between 1 January 2008 and 31 December 2017. Patients having prior allogeneic transplantation, those transplanted in non-EBMT centers, and those receiving double units that were not both released by the Network were excluded. Data related to patients, UCB unit characteristics, and transplant outcomes were collected from the Eurocord and SYRENAD registries in accordance with the ethical standards of the institutional committees and the Helsinki declaration. The European Society for Blood and Marrow Transplantation (EBMT) and the Société Française de Greffe de Moelle et Thérapie Cellulaire (SFGM-TC) approved this study.

\section{Definitions}

The primary endpoint was overall survival (OS) defined as the interval from UCBT to death from any cause. Event-free survival (EFS) was defined as the interval from UCBT to either relapse or death. Transplant-related mortality (TRM) was defined as death in remission. Neutrophil engraftment was defined as the achievement of an absolute neutrophil count $>0.5 \times 10^{9} / \mathrm{L}$ for three consecutive days. Early graft failure was defined as failure to achieve neutrophil engraftment, loss of donor engraftment or autologous recovery within day 100 after HSCT.

Diagnosis and grading of acute and chronic graft versus host diseases (aGVHD and cGVHD) were performed according to standard criteria $[21,22]$. Cell doses were calculated as TNC or $\mathrm{CD} 34^{+}$cell counts by recipient weight in $\mathrm{SCBT}$ or as the cumulative number of $\mathrm{TNC}$ or $\mathrm{CD} 34^{+}$ cells present in both units by recipient weight in dCBT. HLA compatibility was determined considering antigenlevel for HLA-A and -B loci and allele-level for the HLADRB1 locus. HLA compatibility for dCBTs was classified according to the unit with the highest HLA disparity with the recipient. The conditioning regimen was defined as myeloablative (MAC) or reduced intensity (RIC) based on EBMT criteria [23].

\section{Statistical analysis}

Separate analyses were performed for malignant and nonmalignant diseases. The transplant period was analyzed as a binary variable with the cut-off defined by the cohort's median year of UCBT.

Probabilities of OS and EFS were estimated using the Kaplan-Meier method; two-sided log-rank test was used for univariate comparisons. The cumulative incidences were estimated for neutrophil recovery, aGVHD, cGVHD, TRM, and relapse with adjustment for competing risks. Multivariate analyses (MVA) were performed using Cox proportional hazards regression models [24], to adjust for all factors with a $p$ value $<0.10$ in univariate analysis. A threshold level of 0.05 was applied for statistical significance [25]. Statistical analyses were performed using SPSS 21 and R 3.2.0 software packages.

\section{Organization of the Network}

The Network currently includes five active public CBBs and 22 maternity wards authorized to perform UCB collection by the Regional Agency of Health. An additional site dedicated to UCB storage only and five other CBBs that have stopped all collection activities following the Network restructuring, continue to manage and release their previously stored UCB units. The reduction in the total number of active banks and associated maternity wards over the years was part of the global improvement strategy adopted by the network aiming to facilitate the implementation of uniform collection and processing high-quality standards. The processing and storage of UCB units in France can only be performed in public CBBs that are authorized for banking by the National competing authorities. 


\section{Results}

\section{Characteristics of UCB units stored in the Network during the study period}

By the end of 2008, the Network inventory included 7051 UCB units. Due to increasing demand for UCB, the Network increased its activities with the goal of storing 30,000 units by 2015. In 2012, the Network included 16,154 units in its inventory and, in 2015, 34,115 units were available for transplantation. Once the quantitative objective achieved, the Network adopted a qualitative approach based on the "2015 banking criteria" that restricted cryopreservation to units with post-processing TNC counts $\geq 16 \times 10^{8}$. This resulted in the storage of only $10 \%$ of the collected units ( 2076 new UCB units) between 2015 and 2017 (Fig. 1a).

A total of 36,191 UCB units were available in the Network inventory on 31 December $2017 ; 62 \%$ of the stored units had pre-freezing cell counts $>12 \times 10^{8}$ TNC. Only 249 units had TNC counts lower than $6 \times 10^{8}$. All units were typed for HLA -A, -B, and -DRB1 at different resolution levels and $67 \%$ were typed for HLA-C (Table 1).

\section{Characteristics of the UCB units used for transplantation}

Of the 2002 UCB units released by the Network over 10 years, 999 units fulfilled the criteria for inclusion in our study and were used to transplant 796 patients (593 sCBT and $203 \mathrm{dCBT}$ ) in 25 countries and 132 transplant centers (54\% for transplants performed in France). Despite the transient early decrease between 2008 and 2010, the release activity improved dramatically after 2012 reaching a peak in 2014 followed by a slow decrease, which was likely due to the increasing use of related haploidentical donors (Fig. 1b). All released units had intermediate to high-resolution typing for HLA -A, -B, -C, and -DRB1 and 54\% $(n=532)$ had allele-level typing. We observed a sustained increase in the total cell content of the units over the years, most prominent after 2012 (Table 2). A total of 106 units (11\%) had prefreezing TNC counts of $6 \times 10^{8}-12 \times 10^{8}, 193(19 \%)$ had TNC counts of $12 \times 10^{8}-16 \times 10^{8}$, and $709(72 \%)$ had TNC counts $\geq 16 \times 10^{8}$. Only 4 UCB units with TNC count lower than $6 \times 10^{8}$ were released in the 10 -year period considered in the analysis. Volume reduction was performed in $70 \%$ of the units $(n=700)$. UCB used for transplantation were collected between 1995 and 2016 and seventy percent $(n=$ 693) were collected after 2005 (median year of collection). The median pre-freezing cell doses were $3.50 \times 10^{7} \mathrm{TNC} / \mathrm{kg}$ (2.61-5.70) and $1.28 \times 10^{5} \mathrm{CD} 34+$ cells $/ \mathrm{kg}(0.76-2.40)$ for the units collected before 2005 and $4.02 \times 10^{7} \mathrm{TNC} / \mathrm{kg}$ (2.92-6.93) and $1.75 \times 10^{5} \mathrm{CD} 34+$ cells/kg (1.14-3.21) for those collected after 2005 . The median cell loss at transplantation was $23 \%$ (7-49\%) for TNCs and 30\% (3-51\%) for CD34+ cells. This percentage was roughly stable and was not affected by the cryopreservation duration (Table 2). The increased cell counts and available higher resolution HLA typing that characterized the units collected after 2005 might explain the trend towards the enhanced utilization of these units for transplantation in recent years (Fig. 2).

\section{Patient and transplant characteristics}

A total of 796 patients underwent unrelated sCBT ( $n=593$, $75 \%)$ or dCBT $(n=203,25 \%)$ with grafts released by the Network (Table 3). Indications for UCBTs were malignant ( $n=661,83 \%)$ or non-malignant $(n=135,17 \%)$ disorders, with acute leukemia (AL) being the most common diagnosis. Median pre-freezing TNC dose was $5 \times 10^{7} / \mathrm{kg}$ (3.9-6.8) for recipients with hematological malignancies and $12.5 \times 10^{7} / \mathrm{kg}(6.9-21.1)$ for non-malignant disorders. This difference reflects the younger age (and lower bodyweight) of the recipients in the non-malignant group.

\section{Hematological malignancies}

Of the 661 patients with hematological malignancies, $67 \%$ were transplanted for AL (acute lymphoblastic leukemia $(\mathrm{ALL}=168)$; acute myeloblastic leukemia $(\mathrm{AML}=278)$, $17 \%$ for other myeloid malignancies, and $16 \%$ for lymphoproliferative disorders. The median age at UCBT was 35 years $(0.3-74)$ with $35 \%$ of patients aged $<18$ years. Singleunit UCB represented the graft source in $70 \%$ of cases; $87 \%$ of the donor-recipient pairs had $\leq 2 / 6$ HLA disparities (antigen level HLA-A, -B, and allele level DRB1) with the recipients. Allele-level typing for HLA-A, -B, -C, -DRB1 was available for $52 \%(n=162)$ of the patients transplanted before 2012 and 59\% $(n=206)$ of those transplanted after 2012. There was a trend towards selecting UCB units with less HLA mismatches ( $\leq 3 / 8$ HLA disparities in $70 \%)$ and higher cell counts $\left(5.1 \times 10^{7} \mathrm{TNC} / \mathrm{kg}\right)$ for transplants performed after 2012. (Table 3)

Outcomes (Table 4)-The median follow-up for survivors was 36 months (1.9-128). The 60 days cumulative incidence of neutrophil recovery was $88.2 \%$ (95\% CI 85.7-90.7) within a median time of 21 days (5-76), with better engraftment $(90.7 \%$ versus $85.5 \% ; p=0.001)$ after 2012 in comparison to an earlier period. Acute GVHD grade II-IV was observed in 243 patients (54\% of Grade II; $29 \%$ of Grade III and $17 \%$ of Grade IV), within a median of 25 days (7-94) and a cumulative incidence of $36.4 \%$ (95\% CI 32.9-40.3) at 100 days with a trend for higher incidence after $2012(33.1 \%$ vs $40.1 \% p=0.044)$ that might reflect the use of higher cell doses and MAC regimen in recent years; 134 patients developed cGVHD within a median of 


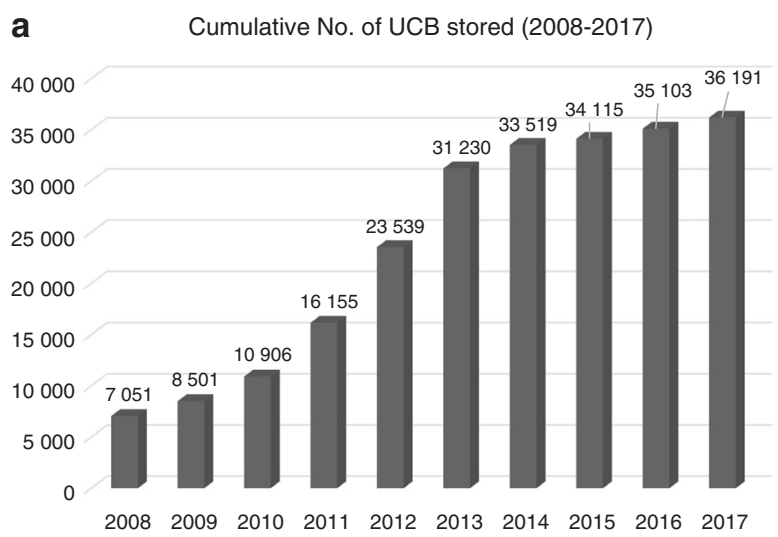

b

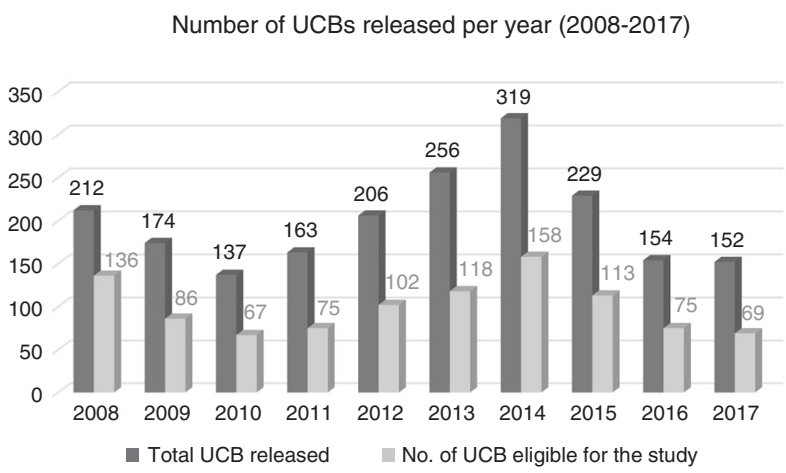

Fig. 1 The Network inventory (2008-2017). a Cumulative number of UCBs stored; b Numbers of UCBs released per year.

Table 1 Characteristics of UCBs available in the Network inventory on 31 December 2008 and 2017.

\begin{tabular}{|c|c|c|c|c|c|c|c|c|}
\hline \multirow[t]{2}{*}{ HLA typing } & \multicolumn{2}{|l|}{ HLA-A } & \multicolumn{2}{|l|}{ HLA-B } & \multicolumn{2}{|l|}{ HLA-C } & \multicolumn{2}{|l|}{ HLA- DRB1 } \\
\hline & 2008 & 2017 & 2008 & 2017 & 2008 & 2017 & 2008 & 2017 \\
\hline Antigen-level & $5485(78 \%)$ & $8117(22 \%)$ & $5408(76 \%)$ & $7691(21 \%)$ & $638(9 \%)$ & $1053(3 \%)$ & $3770(53 \%)$ & $2227(6 \%)$ \\
\hline $\begin{array}{l}\text { Intermediate } \\
\text { resolution (NMDP } \\
\text { code) })^{\mathrm{a}, \mathrm{b}}\end{array}$ & $1566(22 \%)$ & $23,262(65 \%)$ & $1643(24 \%)$ & $23,510(65 \%)$ & $757(11 \%)$ & $16,830(46 \%)$ & $3281(47 \%)$ & $15,995(44 \%)$ \\
\hline Allele-level & - & $4812(13 \%)$ & - & $4990(14 \%)$ & - & $6372(18 \%)$ & - & $17969(50 \%)$ \\
\hline Total UCB typed & $7051(100 \%)$ & $36,191(100 \%)$ & $7051(100 \%)$ & $36,191(100 \%)$ & $1395(20 \%)$ & $24,255(67 \%)$ & $7051(100 \%)$ & $36,191(100 \%)$ \\
\hline
\end{tabular}

$U C B$ umbilical cord blood unit, TNC total nucleated cells.

${ }^{a}$ https://bioinformatics.bethematchclinical.org/hla-resources/allele-codes/.

${ }^{\mathrm{b}}$ Ref. ${ }^{[60]}$.

164 days (70-1015), and an incidence of $25.5 \%$ (95\% CI $22.0-29.6 \%)$ at 3 years. Overall, 321 patients died, 170 of TRM, and 139 of relapse. The cause of death for the remaining 12 patients was unknown. The 3-year incidence of TRM was $31.1 \%$ (95\% CI 27.5-35.1); the most common causes being infection $(n=63)$ and GVHD $(n=42)$. The 3year cumulative incidence of relapse was $23 \%$ (95\% CI 19.7-26.7) with a trend for less relapse in transplants performed after $2012(25.9 \%$ vs $19.7 \% p=0.088)$. The EFS was $46 \% \pm 2$ at 3 years. The OS was $49 \% \pm 3$ at 3 years $(46 \% \pm 3$ before $2012 ; 53 \% \pm 3$ after $2012 ; p=0.24)$ (Fig. 3A).

In MVA, higher pre-freezing TNC dose $\left(>3.5 \times 10^{7} / \mathrm{kg}\right)$ was the only unit characteristic associated with improved OS $[\mathrm{HR}=0.67 ; 95 \% \mathrm{CI}(0.50-0.91) ; p=0.009]$ and lower TRM $[\mathrm{HR}=0.67 ; 95 \%$ CI $(0.46-0.97) ; p=0.03]$; while pre-freezing $\mathrm{CD} 34+$ cell dose $\left(\geq 2.5 \times 10^{6} / \mathrm{kg}\right)$ was predictive of better engraftment $[\mathrm{HR}=1.38 ; 95 \%$ CI $(1.14-1.65) ; p=0.002]$; and higer incidence of aGVHD $[\mathrm{HR}=1.39 ; 95 \% \mathrm{CI}(1.02-1.92) ; p=0.04]$. The transplant period was not predictive of aGVHD. None of the UCB unit characteristics were predictive of relapse or cGVHD.
Importantly, the administration of ATG was the only factor predictive of increased relapse incidence $[\mathrm{HR}=1.47 ; 95 \%$ CI (1.01-2.15); $p=0.04]$ (Table 5).

\section{Clinical results in non-malignant disorders}

UCBT was performed in 135 patients with non-malignant disorders. Indications for transplant were primary immunodeficiency (PID, $n=48$ ), bone marrow failure syndromes (BMF, $n=35$ ), an inborn error of metabolism (IE $n=35$ ), histiocytic disorders (HIS, $n=11$ ), hemoglobinopathy (HGP, $n=5$ ) and autoimmune disease (AID, $n=1$ ). The median age at transplant was $1.6(0.1-43)$ years; only ten patients were older than 18 years. Single UCBT was administered to most patients and MAC regimen was administered to 92 patients. The median infused cell doses were $8.3 \times 10^{7} \mathrm{TNC} / \mathrm{kg}(5.1-15.41)$ and $2.5 \times 10^{5} \mathrm{CD} 34+$ cells/kg (1.3-5.4). All grafts exceeded the minimal cell dose requirements due to the population age and low patients weight. Ninety-three percent of the grafts had $\leq 2 / 6$ HLA disparities with their recipients. Allelic level typing for HLA -A, -B,-C and -DRB1 was available for 59\% $(n=79)$ 
Table 2 Median TNC and CD34+ cell counts of the units released during the study period according to storage year.

\begin{tabular}{|c|c|c|c|c|c|c|c|}
\hline $\begin{array}{l}\text { UCB } \\
\text { storage year }\end{array}$ & $\begin{array}{l}\text { UCB units } \\
\text { released }^{\mathrm{a}} \\
(n)\end{array}$ & $\begin{array}{l}\text { Median } \\
\text { TNC count } \\
\text { at freezing } \\
\left(\times 10^{8}\right)\end{array}$ & $\begin{array}{l}\text { Median } \\
\text { TNC count } \\
\text { at UCBT } \\
\left(\times 10^{8}\right)\end{array}$ & $\begin{array}{l}\text { TNC } \\
\text { loss }(\%)\end{array}$ & $\begin{array}{l}\text { Median } \\
\text { CD34+ } \\
\text { count at } \\
\text { freezing } \\
\left(\times 10^{6}\right)\end{array}$ & $\begin{array}{l}\text { Median } \\
\text { CD34+ } \\
\text { count at } \\
\text { UCBT } \\
\left(\times 10^{6}\right)\end{array}$ & $\begin{array}{l}\text { CD34+ } \\
\text { cell } \\
\text { loss }(\%)\end{array}$ \\
\hline 1995 & 6 & 19.9 & 12.5 & 37 & 7 & 5.2 & 26 \\
\hline 1996 & 4 & 18.4 & 9.4 & 49 & 3.9 & 2.9 & 26 \\
\hline 1997 & 6 & 18.1 & 13.4 & 26 & 4.9 & 3.3 & 33 \\
\hline 1998 & 7 & 16.9 & 13.1 & 22 & 9.9 & 4.9 & 51 \\
\hline 1999 & 48 & 19.1 & 14.7 & 23 & 6.7 & 4 & 40 \\
\hline 2000 & 85 & 18.8 & 14.8 & 21 & 6.1 & 5.1 & 16 \\
\hline 2001 & 64 & 19.4 & 15.1 & 22 & 6 & 3.6 & 40 \\
\hline 2002 & 45 & 18.6 & 13.4 & 28 & 7.4 & 3.6 & 51 \\
\hline 2003 & 32 & 19.1 & 15.4 & 19 & 6.1 & 3.8 & 38 \\
\hline 2004 & 9 & 17.6 & 10.5 & 40 & 5.9 & 5.7 & 3 \\
\hline 2005 & 14 & 17.4 & 11.9 & 32 & 5.4 & 4.5 & 17 \\
\hline 2006 & 29 & 18.1 & 13.1 & 28 & 6.7 & 4.8 & 28 \\
\hline 2007 & 36 & 17.3 & 13.6 & 21 & 6.5 & 4.8 & 26 \\
\hline 2008 & 39 & 19.4 & 15.3 & 21 & 7.5 & 5.3 & 29 \\
\hline 2009 & 53 & 17.0 & 14.5 & 15 & 6.8 & 4.6 & 32 \\
\hline 2010 & 90 & 19.5 & 13.7 & 30 & 8.6 & 5.9 & 31 \\
\hline 2011 & 127 & 19.8 & 15.4 & 22 & 7.8 & 5.3 & 32 \\
\hline 2012 & 142 & 19.8 & 15.3 & 23 & 7.5 & 5.3 & 29 \\
\hline 2013 & 111 & 22.4 & 17.3 & 23 & 8.3 & 5.7 & 31 \\
\hline 2014 & 39 & 24.9 & 19.6 & 21 & 11.7 & 7 & 40 \\
\hline 2015 & 10 & 22.9 & 21.4 & 7 & 7.8 & 6.6 & 15 \\
\hline 2016 & 3 & 25.1 & 21.6 & 14 & 11.6 & 9.7 & 16 \\
\hline
\end{tabular}

${ }^{a}$ Eligible units released during the study period. a UCB units (\%) released per year according to collection period

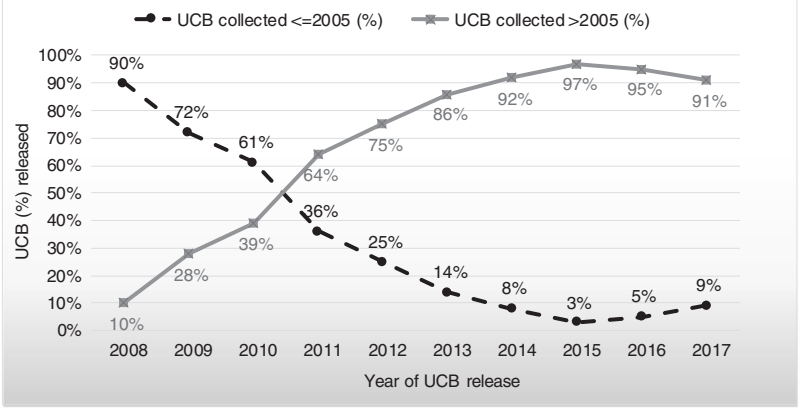

b

UCB $(\%)$ released per year according to collection period

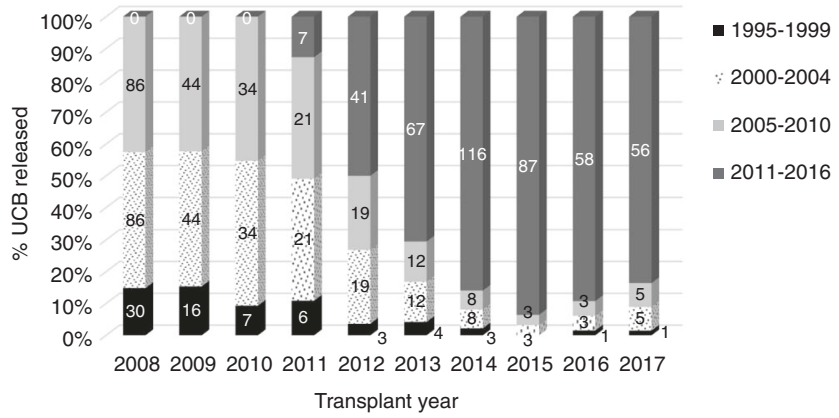

Fig. 2 Percentage of UCB units released yearly. a UCB released according to median year of collection (=<2005 vs $>2005)$; $\mathbf{b}$ UCB released by collection period.

of the patients-UCB pairs with majority $(n=67)$ having $\leq 3$ / 8 disparities (Table 3).

Outcomes (Table 4) - The median follow-up for survivors was 31 (2.9-124) months. The cumulative incidence of neutrophil recovery at day 60 was $79.3 \% \quad(95 \% \mathrm{CI}$ $72.6-86.5 \%$ ) with a median engraftment time of 19 days (6-77) and better engraftment for UCBTs performed after $2012(84.3 \%$ vs $71.2 \% ; p=0.02)$. Grade II-IV aGVHD was reported in 44 patients (22 Grade II, 13 Grade III, and 9 Grade IV) within a median time of 37 days (7-85) and with a cumulative incidence of $33.1 \%$ (95\% CI 25.9-42.2) at 100 days; cGVHD was reported in 21 patients (extensive in five patients) within a median period of 164 days (101-727) and a 3-year incidence of $20.9 \%$ (95\% CI 14.2-30.7). Overall, 47 patients died; the 3-year cumulative incidence of TRM was $34.3 \%$ (95\% CI 27.0-43.5). The most common 
Table 3 Patients and transplant characteristics.

\begin{tabular}{|c|c|c|c|c|}
\hline \multirow[b]{2}{*}{ UCBT period } & \multicolumn{2}{|c|}{ Malignant diseases } & \multicolumn{2}{|c|}{ Non-malignant disorders } \\
\hline & 2008-2012 & 2013-2017 & 2008-2012 & 2013-2017 \\
\hline No. of patients, $N(\%)$ & $313(47 \%)$ & $348(53 \%)$ & $52(39 \%)$ & $83(61 \%)$ \\
\hline Median age, years (IQR) & $35(11-53)$ & $34(9-54)$ & $1.65(0.9-7.7)$ & $1.5(0.7-7)$ \\
\hline Children $(<18 \mathrm{y})$ & $100(32 \%)$ & $133(38 \%)$ & $48(92 \%)$ & $77(93 \%)$ \\
\hline Adults ( $\geq 18 \mathrm{y})$ & $213(68 \%)$ & $215(62 \%)$ & $4(8 \%)$ & $6(7 \%)$ \\
\hline Median weight, kg (IQR) & $60(40-72)$ & $60(31-74)$ & $11(8-25)$ & $11(7-24)$ \\
\hline Gender, Male, $N(\%)$ & $167(53 \%)$ & $189(55 \%)$ & $31(60 \%)$ & $48(58 \%)$ \\
\hline \multicolumn{5}{|l|}{ Recipient CMV serology, $N(\%)$} \\
\hline Negative & $137(46 \%)$ & $151(47 \%)$ & $19(38 \%)$ & $35(47 \%)$ \\
\hline Positive & $161(54 \%)$ & $170(53 \%)$ & $31(62 \%)$ & $39(53 \%)$ \\
\hline Not reported & 15 & 27 & 2 & 9 \\
\hline \multicolumn{5}{|l|}{ Diagnosis, $N(\%)$} \\
\hline Acute leukemias & $209(67 \%)$ & $237(68 \%)$ & & \\
\hline $\begin{array}{l}\text { Myelodysplastic/Myeloproliferative } \\
\text { disorders }\end{array}$ & $42(13 \%)$ & $71(21 \%)$ & & \\
\hline Lymphoproliferative disorders & $49(16 \%)$ & $35(10 \%)$ & & \\
\hline Plasma cell disorders & $13(4 \%)$ & $5(1 \%)$ & & \\
\hline Primary immune deficiency & & & $15(29 \%)$ & $33(40 \%)$ \\
\hline Bone marrow failure syndromes & & & $16(31 \%)$ & $19(23 \%)$ \\
\hline Inborn errors of metabolism & & & $14(27 \%)$ & $21(25 \%)$ \\
\hline Other non-malignant disorders & & & $7(13 \%)$ & $10(12 \%)$ \\
\hline $\begin{array}{l}\text { Time from diagnosis to UCBT, median } \\
\text { months (IQR) }\end{array}$ & $11.5(6-33)$ & $7.86(5-22)$ & $9.16(3.4-20.6)$ & $5(2.6-15)$ \\
\hline \multicolumn{5}{|l|}{ Graft type } \\
\hline sCBT & $215(69 \%)$ & $248(71 \%)$ & $49(94 \%)$ & $81(98 \%)$ \\
\hline $\mathrm{dCBT}$ & $98(31 \%)$ & $100(29 \%)$ & $3(6 \%)$ & $2(2 \%)$ \\
\hline \multicolumn{5}{|l|}{ HLA parity (low-intermediate resolution) } \\
\hline $6 / 6$ & $17(5 \%)$ & $23(8 \%)$ & $6(12 \%)$ & $18(22 \%)$ \\
\hline $5 / 6$ & $93(31 \%)$ & $126(43 \%)$ & $37(71 \%)$ & $41(49 \%)$ \\
\hline $4 / 6$ & $183(59 \%)$ & $131(45 \%)$ & $8(15 \%)$ & $15(18 \%)$ \\
\hline $3 / 6$ & $13(4 \%)$ & $12(4 \%)$ & $1(2 \%)$ & 0 \\
\hline $2 / 6$ & $2(1 \%)$ & 0 & 0 & 0 \\
\hline Missing & 5 & 56 & 0 & $9(11 \%)$ \\
\hline \multicolumn{5}{|l|}{ HLA parity (high resolution) } \\
\hline $8 / 8$ & $2(1 \%)$ & $6(3 \%)$ & $1(4 \%)$ & $10(19 \%)$ \\
\hline $7 / 8$ & $7(4 \%)$ & $26(13 \%)$ & $5(19 \%)$ & $11(21 \%)$ \\
\hline $6 / 8$ & $26(16 \%)$ & $55(27 \%)$ & $6(23 \%)$ & $17(32 \%)$ \\
\hline $5 / 8$ & $64(40 \%)$ & $56(27 \%)$ & $8(31 \%)$ & $9(17 \%)$ \\
\hline $4 / 8$ & $42(26 \%)$ & $52(25 \%)$ & $5(19 \%)$ & $3(6 \%)$ \\
\hline $3 / 8$ & $18(11 \%)$ & $10(5 \%)$ & $1(4 \%)$ & $3(6 \%)$ \\
\hline $2 / 8$ & $3(2 \%)$ & $1(<1 \%)$ & & \\
\hline \multicolumn{5}{|l|}{ ABO compatibility, $N(\%)$} \\
\hline ABO compatible & $117(38 \%)$ & $120(36 \%)$ & $24(46 \%)$ & $31(38 \%)$ \\
\hline Minor ABO Incompatibility & $80(26 \%)$ & $76(23 \%)$ & $12(23 \%)$ & $18(22 \%)$ \\
\hline Major ABO Incompatibility & $112(36 \%)$ & $135(41 \%)$ & $16(31 \%)$ & $32(40 \%)$ \\
\hline Missing & 4 & 17 & 0 & 2 \\
\hline \multicolumn{5}{|l|}{ Gender compatibility, $N(\%)$} \\
\hline $\mathrm{M} / \mathrm{M}$ or $\mathrm{F} / \mathrm{F}$ & $126(40 \%)$ & $160(47 \%)$ & $26(50 \%)$ & $44(54 \%)$ \\
\hline $\mathrm{M} / \mathrm{F}$ or $\mathrm{F} / \mathrm{M}$ & $187(60 \%)$ & $182(53 \%)$ & $26(50 \%)$ & $38(46 \%)$ \\
\hline Missing & & 6 & 0 & 1 \\
\hline \multicolumn{5}{|l|}{ Conditioning regimen } \\
\hline MAC & $164(53 \%)$ & $223(66 \%)$ & $32(63 \%)$ & $60(75 \%)$ \\
\hline RIC & $146(47 \%)$ & $115(34 \%)$ & $19(37 \%)$ & $20(25 \%)$ \\
\hline Not reported & 3 & 10 & 1 & 3 \\
\hline TBI containing regimen & $186(59 \%)$ & $141(41 \%)$ & $8(16 \%)$ & $12(20 \%)$ \\
\hline ATG administration & $149(49 \%)$ & $145(62 \%)$ & $39(77 \%)$ & $66(93 \%)$ \\
\hline
\end{tabular}




\begin{tabular}{|c|c|c|c|c|}
\hline \multirow[b]{2}{*}{ UCBT period } & \multicolumn{2}{|c|}{ Malignant diseases } & \multicolumn{2}{|c|}{ Non-malignant disorders } \\
\hline & 2008-2012 & 2013-2017 & 2008-2012 & 2013-2017 \\
\hline \multicolumn{5}{|l|}{ GVHD Prophylaxis } \\
\hline $\mathrm{CSA} \pm \mathrm{MMF}$ based & $300(96 \%)$ & $305(88 \%)$ & $45(87 \%)$ & $74(89 \%)$ \\
\hline Others & $13(4 \%)$ & $43(12 \%)$ & $7(13 \%)$ & $9(11 \%)$ \\
\hline \multicolumn{5}{|l|}{ UCB characteristics } \\
\hline - UCB median volume, ml (IQR) & $136(117-163)$ & $162(140-183)$ & $124(108-143)$ & $145(126-168)$ \\
\hline - UCB volume reduction, $n(\%)$ & $191(46 \%)$ & $410(92 \%)$ & $23(43 \%)$ & $76(89 \%)$ \\
\hline \multicolumn{5}{|l|}{ Cell dose, Median (IQR) } \\
\hline - TNC collected $\left(\times 10^{7} / \mathrm{kg}\right)$ & $4.8(3.8-6.5)$ & $5.1(3.9-7.2)$ & $11(7.0-20.1)$ & $12.9(6.9-21.1)$ \\
\hline - $\mathrm{CD} 34$ collected $\left(\times 10^{5} / \mathrm{kg}\right)$ & $1.9(1.3-2.8)$ & $2.3(1.5-3.4)$ & $4.3(2.3-9.3)$ & $4.5(2.5-7.9)$ \\
\hline - TNC infused $\left(\times 10^{7} / \mathrm{kg}\right)$ & $3.7(2.9-5.3)$ & $4.1(3.0-6.1)$ & $8.3(5.1-15.41)$ & $9.2(4.8-15.1)$ \\
\hline - CD34 infused $\left(\times 10^{5} / \mathrm{kg}\right)$ & $1.4(1.0-2.3)$ & $1.7(1.1-2.5)$ & $2.5(1.3-5.4)$ & $3.1(1.5-5.5)$ \\
\hline Follow-up, median months (range) & $65.8(1.9-128)$ & $23.6(2.5-67)$ & $74(3.4-124)$ & $25(2.9-60)$ \\
\hline
\end{tabular}

$U C B T$ cord blood transplant, $I Q R$ interquartile range $(\mathrm{Q} 1-\mathrm{Q} 3)$, $s C B T$ single unit UCBT, $d C B T$ double unit UCBT, $M A C$ myeloablative conditioning, RIC reduced intensity conditioning, TBI total body irradiation, $A T G$ anti-thymocyte globulins, GVHD graft versus host disease, $C S A$ ciclosporine A, $M M F$ mycophenolate mefotil, $U C B$ umbilical cord blood unit, $T N C$ total nucleated cells.

causes of TRM were infection $(n=17)$ and $\operatorname{GVHD}(n=8)$. Twenty-seven patients developed primary graft failure, 19 died in a median time of 3.9 months, seven patients were alive at last follow-up after a second allogeneic transplant, and one patient after autologous reconstitution. Three-year OS was 59\% \pm 6 for patients transplanted before 2012 and $65 \% \pm 5$ for those after 2012 (Fig. 3B) with large heterogeneity among diagnosis (49\% for BMF, $53 \%$ for HIS, and $75 \%$ for both IE and HbP). In MVA (Table 5), positive recipient CMV serology was the only factor associated with adverse OS $[\mathrm{HR}=2.66 ; 95 \% \mathrm{CI}(1.36-5.19) ; p=0.004]$ and TRM $[\mathrm{HR}=2.76 ; 95 \%$ CI $(1.37-5.55) ; p=0.004]$. Other factors did not show any significant impact on transplant outcomes.

\section{Discussion}

The first successful UCBT was performed in 1988 at SaintLouis hospital in Paris [1] in a patient with Fanconi anemia who received an UCB unit from his HLA-identical sibling. Subsequently, encouraging outcomes of HLA-matched sibling UCBTs provided the proof-of-concept that UCB was a good alternative source of hematopoietic stem cells. The first promising unrelated UCBT results were reported shortly after in children [26-29] and adults, promoted by improved patient and UCB unit selection and better supportive care [3, 4].

The first French CBB was set in Paris in 1990 and started collecting UCB units and developing its registry. With the growing evidence that UCB units could be stored without compromising their biological properties [30] and the acceptance of UCB as an alternative stem cell source, several public institutions, started to create CBBs in the US [31, 32] and Europe [33-37].
Subsequently, an international cooperative CBB network, the NetCord group, was created in 1998 to ensure the high and uniform quality of all UCB units [38]. The group promoted the creation of registries, such as Eurocord, to document and evaluate transplant outcomes in recipients of units released by CBBs.

According to the World Marrow Donors Association (WMDA) reports [39], more than 790,000 UCB units are available worldwide in the public CBBs and more than 40,000 UCBTs have been performed to date [40]. Such numbers highlight the significant clinical achievements over the last 30 years which established UCB as an alternative stem cell source for transplantation in children and adults. However, the use of new transplant protocols involving related haploidentical donors in recent years, has led to a dramatic decrease in utilization of UCB worldwide, thus challenging the sustainability of the public CBB economic model. Many publications addressing this issue advised the CBBs to re-calculate the size of their inventory defining banking decisions based on higher thresholds for cell counts and stronger quality criteria [41-43].

Despite setting up its first bank in 1990, the French Network had stored only 7051 allogeneic UCB units in three operational CBBs by the end of 2008. Adapted from the French bone marrow registry model [44], the optimal size for the French Network was estimated to include 46,737 CBU, which would provide national patients an $83 \%$ chance of finding a compatible CBU. To respond to the increase in demand for UCB grafts by transplant centers and to reduce the costly dependence on imported UCB units, the Network, supported by the French health authorities, set a target to store $30,000 \mathrm{UCB}$ units by 2015 , then adopted in 2015 a qualitative approach for banking UCB based on higher thresholds for cell counts (prefreezing $\left.\mathrm{TNC}>16 \times 10^{8}\right)$. More importantly, the Network imposed 
Table 4 Outcomes for malignant and non-malignant diseases by transplant period.

\begin{tabular}{|c|c|c|c|c|}
\hline & Total & $2008-2012$ & 2013-2017 & $P$ value \\
\hline \multicolumn{5}{|l|}{3 y Overall survival } \\
\hline Malignant diseases & $49 \% \pm 3$ & $46 \% \pm 3$ & $53 \% \pm 3$ & 0.241 \\
\hline Non-malignant disorders & $62 \% \pm 4$ & $59 \% \pm 6$ & $65 \% \pm 5$ & 0.500 \\
\hline \multicolumn{5}{|l|}{3 y Event-free survival } \\
\hline Malignant diseases & $46 \% \pm 2$ & $42 \% \pm 3$ & $50 \% \pm 3$ & 0.108 \\
\hline Non-malignant disorders & NA & NA & NA & \\
\hline \multicolumn{5}{|l|}{ d60 CI Engraftment } \\
\hline Malignant diseases & $88.2 \%(85.7-90.7)$ & $85.5 \%(81.7-89.6)$ & $90.7 \%(87.7-93.8)$ & 0.001 \\
\hline Non-malignant disorders & $79.3 \%(72.6-86.5)$ & $71.2 \%(59.6-85.0)$ & $84.3 \%(76.7-92.7)$ & 0.02 \\
\hline \multicolumn{5}{|l|}{ d100 CI aGVHD } \\
\hline Malignant diseases & $36.4 \%(32.9-40.3)$ & $33.1 \%(28.2-38.8)$ & $40.1 \%(35.2-45.7)$ & 0.044 \\
\hline Non-malignant disorders & $33.1 \%(25.9-42.2)$ & $35.3 \%(24.2-51.4)$ & $31.7 \%(23.0-43.7)$ & 0.736 \\
\hline \multicolumn{5}{|l|}{3 y CI cGVHD } \\
\hline Malignant diseases & $25.5 \%(22.0-29.6)$ & $26.0 \%(21.1-32.2)$ & $24.8 \%(20.1-30.5)$ & 0.968 \\
\hline Non-malignant disorders & $20.9 \%(14.2-30.7)$ & $20.9 \%(11.2-39.1)$ & $15.2 \%(8.5-27.0)$ & 0.585 \\
\hline \multicolumn{5}{|l|}{3 y CI relapse } \\
\hline Malignant diseases & $23.0 \%(19.7-26.7)$ & $25.9 \%(21.3-31.6)$ & $19.7 \%(15.7-24.9)$ & 0.088 \\
\hline Non-malignant disorders & NA & NA & NA & \\
\hline \multicolumn{5}{|l|}{3 у CI TRM } \\
\hline Malignant disorders & $31.1 \%(27.5-35.1)$ & $31.8 \%(26.8-37.7)$ & $30.1 \%(25.3-35.7)$ & 0.861 \\
\hline Non-malignant disorders & $34.3 \%(27.0-43.5)$ & $37.9 \%(26.4-54.3)$ & $31.7 \%(23.0-43.7)$ & 0.495 \\
\hline
\end{tabular}

Statistically significant $p$-values are in bold italic and Statistically non-significant $p$-values are in italic. a Malignant diseases

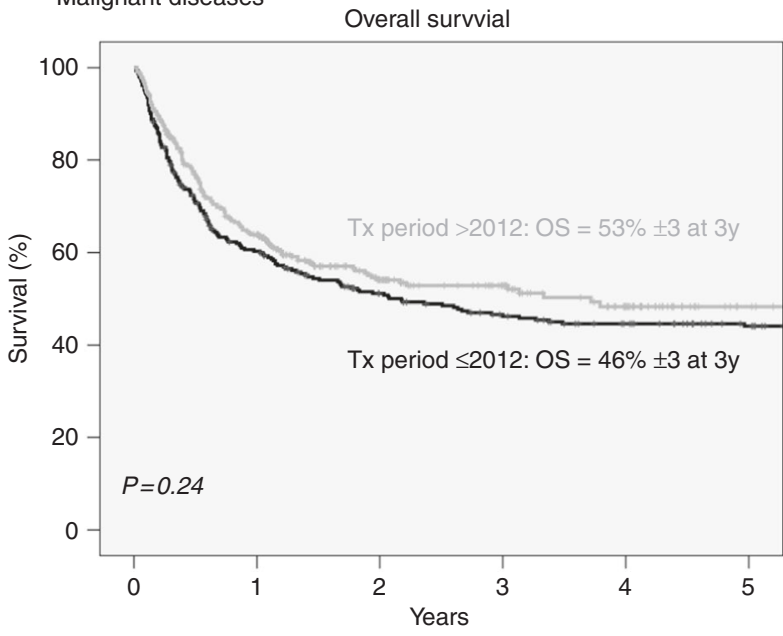

b Non-malignant

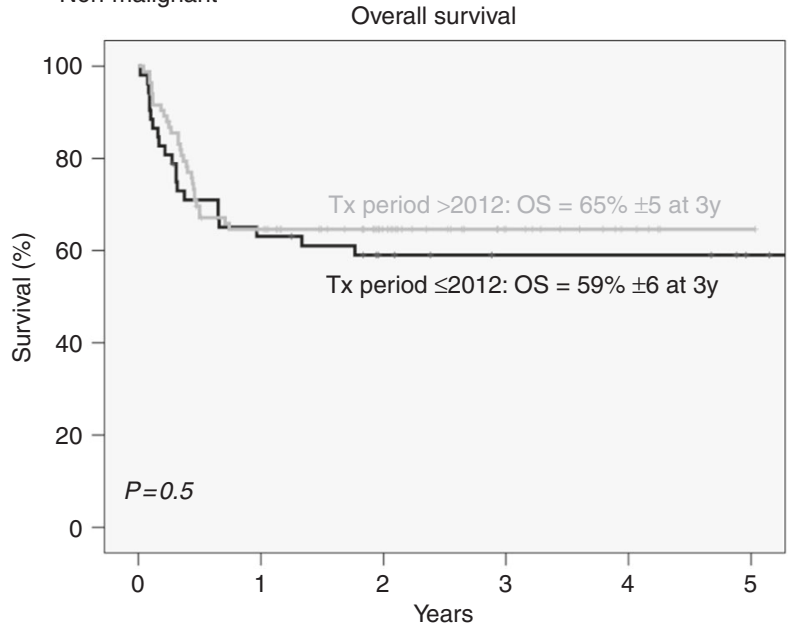

Fig. 3 Probabilities of 3-year overall survival by transplant period. a OS for malignant diseases and b OS for non-malignant disorders.

very stringent quality standards for collection procedures. These standards used to be regularly modified and updated in the context of a quality improvement process using recipients' outcomes results provided yearly by Eurocord. Consequently, on 31 December 2017, more than $36 \%$ of the units in the Network inventory had a cell content exceeding $15 \times 10^{8}$ TNCs, compared to only $20 \%$ of the units listed worldwide in WMDA 2018 reports [39], underscoring a successful quality improvement process adopted by the Network.
In our study, we report 999 UCB units collected and released by the Network for transplantation in EBMT centers. The units collected in recent years were characterized by higher cell counts and allele-level HLA typing which translated into an enhanced utilization rate and a shorter storage period, mainly for those collected after 2015 . When compared to current unit selection guidelines [5, 15, 18, 45, 46], 98\% of the donor-recipient pairs had 0-2/ 6 HLA disparities at antigen level HLA- A, -B and allele- 
Table 5 Multivariate analysis for overall survival, engraftment, aGVHD, cGVHD, TRM and relapse by disease type.

\begin{tabular}{|c|c|c|c|c|}
\hline \multicolumn{5}{|l|}{ Malignant diseases } \\
\hline \multirow[t]{2}{*}{ Covariates $^{\mathrm{a}}$} & \multirow[t]{2}{*}{ HR } & \multicolumn{2}{|c|}{$95 \% \mathrm{CI}$} & \multirow[t]{2}{*}{$p$ value } \\
\hline & & Lower & Higher & \\
\hline \multicolumn{5}{|l|}{ Overall survival } \\
\hline Recipent CMV (positive vs negative) & 1.32 & 1.04 & 1.66 & 0.020 \\
\hline Age $(\geq 18$ y vs $<18$ y) & 1.59 & 1.18 & 2.15 & 0.002 \\
\hline Pre-cryopreservation TNC ( $\geq 3.5$ vs $<3.5$ ) & 0.67 & 0.50 & 0.91 & 0.009 \\
\hline \multicolumn{5}{|l|}{ PMN engraftment } \\
\hline UCBT period (>2012 vs $\leq 2012$ ) & 1.20 & 1.01 & 1.43 & 0.040 \\
\hline Pre-cryopreservation CD34 ( $\geq 2.5$ vs $<2.5$ ) & 1.38 & 1.14 & 1.65 & 0.0016 \\
\hline \multicolumn{5}{|l|}{ aGVHD } \\
\hline RIC vs MAC & 0.63 & 0.45 & 0.89 & 0.008 \\
\hline ATG vs No ATG & 0.31 & 0.19 & 0.50 & $<0.001$ \\
\hline Pre-cryopreservation CD34 ( $\geq 2.5$ vs $<2.5)$ & 1.39 & 1.02 & 1.92 & 0.040 \\
\hline \multicolumn{5}{|l|}{ cGVHD } \\
\hline dCBU vs sCBU & 1.34 & 0.95 & 1.91 & 0.098 \\
\hline \multicolumn{5}{|l|}{ TRM } \\
\hline Recipient CMV (positive vs negative) & 1.49 & 1.10 & 2.03 & 0.010 \\
\hline Age $(\geq 18 y$ vs $<18 y)$ & 2.24 & 1.47 & 3.39 & $<0.001$ \\
\hline Pre-cryopreservation TNC ( $\geq 3.5$ vs $<3.5)$ & 0.67 & 0.46 & 0.97 & 0.030 \\
\hline \multicolumn{5}{|l|}{ RI } \\
\hline ATG vs No ATG & 1.47 & 1.01 & 2.15 & 0.040 \\
\hline \multicolumn{5}{|l|}{ Event-free survival } \\
\hline Recipent CMV (positive vs negative) & 1.35 & 1.06 & 1.73 & 0.020 \\
\hline Age $(\geq 18$ y vs $<18 y)$ & 1.40 & 1.02 & 1.93 & 0.030 \\
\hline No $C R$ vs $C R$ & 1.51 & 1.14 & 1.99 & 0.004 \\
\hline TBI vs no TBI & 0.67 & 0.52 & 0.87 & 0.003 \\
\hline
\end{tabular}

Non-malignant disorders

Overall survival

$\begin{array}{lllll}\text { Recipient CMV (positive vs negative) } & 2.66 & 1.36 & 5.19 & \mathbf{0 . 0 0 4}\end{array}$

$\begin{array}{lllll}\text { Median age }(\geq 1.5 \mathrm{y} \text { vs }<1.5) & 1.80 & 0.95 & 3.38 & 0.077\end{array}$

PMN engraftment

$\begin{array}{lllll}\text { UCBT period }(>2012 \text { vs } \leq 2012) & 1.38 & 0.90 & 2.10 & 0.130 \\ \text { Pre-cryopreservation CD34 }(\geq 3 \text { vs }<3) & 1.240 & 0.76 & 2.03 & 0.611\end{array}$
aGVHD

No significant factor

cGVHD

\begin{tabular}{lllll} 
Median age $(\geq 1.5 \mathrm{y}$ vs $<1.5 \mathrm{y})$ & 2.39 & 0.67 & 8.54 & 0.180 \\
RIC vs MAC & 2.15 & 0.81 & 5.72 & 0.126 \\
Pre-cryopreservation CD34 ( $\geq 3$ vs $<3)$ & 0.32 & 0.08 & 1.33 & 0.119 \\
Recipient CMV (positive vs negative) & 2.76 & 1.37 & 5.55 & $\mathbf{0 . 0 0 4}$ \\
\hline
\end{tabular}

Statistically significant $p$-values are in bold italic and Statistically non-significant $p$-values are in italic. $a G V H D$ acute graft vs host disease, $A T G$ anti-tymocyte globulins, $c G V H D$ chronic graft vs host disease, $C M V$ cytomegalovirus, $C R$ complete remission, $d C B T$ double cord blood transplant, $M A C$ myeloablative conditioning, $P M N$ polymorphonuclear cells, $R I$ relapse incidence, $R I C$ reduced intensity conditioning, $s C B T$ single-unit cord blood transplant, TBI total body irradiation, TNC total nucleated cells, TRM transplantrelated mortality, UCBT umbilical cord blood transplant.

${ }^{\mathrm{a}}$ Covariates included in the multivariate model: transplant period, age, recipient $\mathrm{CMV}$, myolablative nature of the conditioning regimen, TBI, ATG, remission status, cell dose and graft type. 
level HLA_DRB1) and 52\% had 0-4/8 allele level HLA mismatches. In addition, $86 \%$ of the patients received a prefreezing cell dose exceeding the minimum requirement for TNC $\left(>3.5 \times 10^{7} / \mathrm{kg}\right)$, and $75 \%$ received a $\mathrm{CD} 34+$ cell dose $\geq 1.5 \times 10^{5} / \mathrm{kg}$, with higher cell doses in the nonmalignant setting reflecting younger population age and lower body weight. The median TNC loss after thawing was $23 \%$ and was not affected by the length of storage or the processing $\mathrm{CBB}$, demonstrating well-standardized methods of cryopreservation across the Network through time. UCBTs performed after 2012 were significantly associated with faster neutrophil recovery both for malignant $(90.7 \%$ vs $85.5 \%, p=0.001)$ and non-malignant disorders $(84.3 \%$ vs $71.2 \%, p=0.02)$ reflecting improved selection criteria for patients and UCB units in recent years.

As previously reported by other groups, we were not able to detect a clear association of HLA disparity with acute $[2,29]$ or chronic GVHD [10, 47, 48] occurrence, although $46 \%$ of the transplants had $\geq 2 / 6$ HLA mismatches.

The reduced incidence of relapse $(25 \%$ relapse at 3 years) in our cohort was broadly similar to that reported in hematological malignancies $[15,49,50]$ with significantly higher rates in ATG-recipients $(p=0.04)$. The incidence of TRM was $33.1 \%$ for malignant and $34.3 \%$ for nonmalignant disorders and did not improve overtime despite better unit selection. The OS rate was $49 \% \pm 4$ in hematological malignancies and $62 \pm 4 \%$ in non-malignant disorders and compares favorably with previously published results for UCBT. When looking at MVA of outcomes in malignant diseases, cell dose was the only unit-related factor for OS, EFS, engraftment, acute GVHD, and TRM indicating that improvement could be obtained by further increase of the cell dose, especially in adults. None of the unit-related factors was predictive of outcomes in the nonmalignant disorders probably due to younger age and higher cell counts.

Despite lower release rates in recent years with the emergence of haploidentical transplants, $6 \%$ of the network inventory were released over the 10-year study period and $2.7 \%$ were exported (2\% after 2012), reflecting the highquality standards of the French banked units and their probable HLA diversity. This high release activity represented a significant source of income for the Network close to $€ 8500$ per unit transplanted nationally and $€ 17,000$ per exported unit.

Based on the data from the WMDA reports [39], France maintained the greatest utilization rates $(0.3-0.4 \%$ of the Network inventory) in Europe over the last 10 years and the highest ratio of exporting units $(0.1 \%)$, promoted by highquality of the units facilitated by the Network, and an exportation fee $15-20 \%$ lower than the average fee charged by other countries $(€ 20,000-€ 25,000)$.
Our study has limitations inherent to its retrospective registry-based nature and the heterogenous group of diseases included. Another limitation is the consequence of the unavailability of donor's ethnical background data due to restrictions imposed by the French legislation. Consequently, the analysis of the diversity of HLA haplotypes available in the network inventory (including rare and ethnic minorities) could not be performed. Finally, with the very strict and ambitious criteria for selecting the units eligible to be stored, the Network had to regularly address the economic challenges to remain viable and increase the utilization rates of the units available in its inventory.

Like the French Network, many National banks around the world $[5,51-58]$ started to question how to reach the optimal size of their inventory in the best cost-effective way and to reorganize their CBB networks to become economically sustainable without compromising the probability of finding optimal grafts for their patients. Consequently, the 2018 WMDA report [39] summarizing trends in stem cell registries in Europe, showed dramatic decrease in the numbers of new banked unrelated UCB units, from 90,000 new CB units listed worldwide in 2010 to less than half $(40,000)$ in 2015, and 10,000 in December 2017.

In conclusion, despite the obvious decrease in the use of CBT in the latest years, with the development of haploidentical transplants, UCB remains an important source of stem cells mainly in children and for patients belonging to ethnic minorities [56, 59]. The Network still has to face two major challenges: expanding the diversity of HLA profiles represented in its inventory and achieving financial sustainability while maintaining the same or improved quality standards. Because all cryopreserved units are tested and ready for immediate use, UCB is also an invaluable resource in urgent situations as in the recent COVID-19 epidemics which resulted in a $15 \%$ increase in the number of units released by the Network to transplant national recipients over 2020 (82 units from January through October 2020 compared to 71 in the same period in 2019). Novel approaches aiming to speed-up hematopoietic recovery using ex-vivo expansion systems for hematopoietic stem cells and innovative cell and gene therapies using cord blood-derived immune cells (CAR, NK, MSC) are still being explored for the treatment of hematological diseases as well as autoimmune, neurological, and inflammatory disorders, thus determining the beginning of a new era in UCB therapy.

Acknowledgements We would like to thank Dr. Sergi Querol for his assistance in accessing data from the Antony Nolan and REDMO registries and to acknowledge the work of Ms Cristina Fusté (Data manager at REDMO, Spanish Bone Marrow Registry), and $\mathrm{Mr}$ Mahamadou Sinayako (Biostatistician at the ABM, SYRENAD Registry) who have provided the data. 


\section{Compliance with ethical standards}

Conflict of interest The authors declare no competing interests.

Publisher's note Springer Nature remains neutral with regard to jurisdictional claims in published maps and institutional affiliations.

\section{References}

1. Gluckman E, Broxmeyer HE, Auerbach AD, Friedman HS, Douglas GW, Devergie A, et al. Hematopoietic reconstitution in a patient with Fanconi's anemia by means of umbilical-cord blood from an HLA-identical sibling. N. Engl J Med. 1989;321:1174-8.

2. Gluckman E, Rocha V. Cord blood transplantation: state of the art. Haematologica. 2009;94:451-4.

3. Gluckman E, Ruggeri A, Volt F, Cunha R, Boudjedir K, Rocha V. Milestones in umbilical cord blood transplantation. Br J Haematol. 2011;154:441-7.

4. Ballen KK, Gluckman E, Broxmeyer HE. Umbilical cord blood transplantation: the first 25 years and beyond. Blood. 2013;122:491-498.

5. Barker JN, Kurtzberg J, Ballen K, Boo M, Brunstein C, Cutler C, et al. Optimal practices in unrelated donor cord blood transplantation for hematologic malignancies. Biol Blood Marrow Transpl. 2017;23:882-96.

6. Rocha V, Wagner JE, Sobocinski KA, Klein JP, Zhang M-J, Horowitz MM, et al. Graft-versus-host disease in children who have received a cord-blood or bone marrow transplant from an hla-identical sibling. N. Engl J Med. 2000;342:1846-54.

7. Rocha V, Labopin M, Sanz G, Arcese W, Schwerdtfeger R, Bosi A, et al. Transplants of umbilical-cord blood or bone marrow from unrelated donors in adults with acute leukemia. N. Engl J Med. 2004;351:2276-85.

8. Weisdorf D, Eapen M, Ruggeri A, Zhang M-J, Zhong X, Brunstein $\mathrm{C}$, et al. Alternative donor transplantation for older patients with acute myeloid leukemia in first complete remission: a center for international blood and marrow transplant researcheurocord analysis. Biol Blood Marrow Transpl. 2014;20:816-22.

9. Ruggeri A, Sanz G, Bittencourt H, Sanz J, Rambaldi A, Volt F, et al. Comparison of outcomes after single or double cord blood transplantation in adults with acute leukemia using different types of myeloablative conditioning regimen, a retrospective study on behalf of Eurocord and the Acute Leukemia Working Party of EBMT. Leukemia. 2014;28:779-86.

10. Laughlin MJ, Eapen M, Rubinstein P, Wagner JE, Zhang M-J, Champlin RE, et al. Outcomes after transplantation of cord blood or bone marrow from unrelated donors in adults with leukemia. $\mathrm{N}$. Engl J Med. 2004;351:2265-75.

11. Brunstein CG, Fuchs EJ, Carter SL, Karanes C, Costa LJ, Wu J, et al. Alternative donor transplantation after reduced intensity conditioning: results of parallel phase 2 trials using partially HLAmismatched related bone marrow or unrelated double umbilical cord blood grafts. Blood. 2011;118:282-8.

12. Rocha V, Gluckman E, on behalf of the Eurocord-Netcord registry and European Blood and Marrow Transplant group. Improving outcomes of cord blood transplantation: HLA matching, cell dose and other graft- and transplantation-related factors. Br J Haematol. 2009;147:262-74.

13. Barker JN, Scaradavou A, Stevens CE. Combined effect of total nucleated cell dose and HLA match on transplantation outcome in 1061 cord blood recipients with hematologic malignancies. Blood. 2010;115:1843-9.
14. Eapen M, Klein JP, Sanz GF, Spellman S, Ruggeri A, Anasetti C, et al. Effect of donor-recipient HLA matching at HLA A, B, C, and DRB 1 on outcomes after umbilical-cord blood transplantation for leukaemia and myelodysplastic syndrome: a retrospective analysis. Lancet Oncol. 2011;12:1214-21.

15. Eapen M, Klein JP, Ruggeri A, Spellman S, Lee SJ, Anasetti C, et al. Impact of allele-level HLA matching on outcomes after myeloablative single unit umbilical cord blood transplantation for hematologic malignancy. Blood. 2014;123:133-40.

16. Eapen M, Wang T, Veys PA, Boelens JJ, St Martin A, Spellman $S$, et al. Allele-level HLA matching for umbilical cord blood transplantation for non-malignant diseases in children: a retrospective analysis. Lancet Haematol. 2017;4:e325-33.

17. Ruggeri A. Optimizing cord blood selection. Hematology. 2019;2019:522-31.

18. Dehn J, Spellman S, Hurley CK, Shaw BE, Barker JN, Burns LJ, et al. Selection of unrelated donors and cord blood units for hematopoietic cell transplantation: guidelines from the NMDP/ CIBMTR. Blood. 2019;134:924-34.

19. Étude de législation comparée $\mathrm{n}^{\circ} 187$ - septembre 2008 - La conservation du sang placentaire. https://www.senat.fr/lc/lc187/ lc187_mono.html. 2008.

20. International standards for cord blood collection, banking and release for administration, accreditation manual. http://www.factw ebsite.org/uploadedFiles/Standards/NetCord\%20FACT\%206th\% 20Ed\%20Manual\%20Draft.09.01.15.pdf.

21. Przepiorka D, Weisdorf D, Martin P, Klingemann HG, Beatty P, Hows J, et al. 1994 Consensus Conference on Acute GVHD Grading. Bone Marrow Transplant. 1995;15:825-8.

22. Martin PJ, Weisdorf D, Przepiorka D, Hirschfeld S, Farrell A, Rizzo JD, et al. National Institutes of Health Consensus Development Project on Criteria for Clinical Trials in Chronic Graftversus-Host Disease: VI. Design of Clinical Trials Working Group Report. Biol Blood Marrow Transpl. 2006;12:491-505.

23. Spyridonidis A, Labopin M, Savani BN, Niittyvuopio R, Blaise D, Craddock $\mathrm{C}$, et al. Redefining and measuring transplant conditioning intensity in current era: a study in acute myeloid leukemia patients. Bone Marrow Transplant. 2020; https://doi.org/10. 1038/s41409-020-0803-y.

24. Cox DR. Regression models and life-tables. J R Stat Soc Ser B Methodol. 1972;34:187-220.

25. Gooley TA, Leisenring W, Crowley J, Storer BE. Estimation of failure probabilities in the presence of competing risks: new representations of old estimators. Stat Med. 1999;18:695-706.

26. Wagner JE, Broxmeyer HE, Byrd RL, Zehnbauer B, Schmeckpeper B, Shah N, et al. Transplantation of umbilical cord blood after myeloablative therapy: analysis of engraftment. Blood. 1992;79:1874-81.

27. Wagner JohnE, Steinbuch M, Kernan NancyA, Broxmayer HalE, Gluckman E. Allogeneic sibling umbilical-cord-blood transplantation in children with malignant and non-malignant disease. Lancet. 1995;346:214-9.

28. Kurtzberg J, Laughlin M, Graham ML, Smith C, Olson JF, Halperin EC, et al. Placental blood as a source of hematopoietic stem cells for transplantation into unrelated recipients. N. Engl J Med. 1996;335:157-66.

29. Rubinstein P, Carrier C, Scaradavou A, Kurtzberg J, Adamson J, Migliaccio AR, et al. Outcomes among 562 recipients of placental-blood transplants from unrelated donors. N. Engl J Med. 1998;339:1565-77.

30. Broxmeyer HE, Lee M-R, Hangoc G, Cooper S, Prasain N, Kim Y-J, et al. Hematopoietic stem/progenitor cells, generation of induced pluripotent stem cells, and isolation of endothelial progenitors from 21- to 23.5-year cryopreserved cord blood. Blood. 2011;117:4773-7. 
31. Rubinstein P, Dobrila L, Rosenfield RE, Adamson JW, Migliaccio G, Migliaccio AR, et al. Processing and cryopreservation of placental/umbilical cord blood for unrelated bone marrow reconstitution. Proc Natl Acad Sci USA. 1995;92:10119-22.

32. Rubinstein P, Taylor PE, Scaradavou A, Adamson JW, Migliaccio G, Emanuel D, et al. Unrelated placental blood for bone marrow reconstitution: organization of the placental blood program. Blood Cells. 1994;20:587-96 ; discussion 596-600.

33. Navarrete C, Contreras M. Cord blood banking: a historical perspective. Br J Haematol. 2009;147:236-45.

34. Lazzari L, Corsini C, Curioni C, Lecchi L, Scalamogna M, Rebulla P, et al. The Milan Cord Blood Bank and the Italian Cord Blood Network. J Hematother. 1996;5:117-22.

35. Navarrete $C$, Warwick R, Armitage S, Fehily D, Contreras M. The London Cord Blood Bank. Bone Marrow Transpl. 1998;22:S6-7.

36. Querol S, Gabarró M, Amat L, Gonzalez S, Gomez MD, de la Calle $\mathrm{O}$, et al. The placental blood program of the Barcelona Cord Blood Bank. Bone Marrow Transpl. 1998;22:S3-5.

37. Dal Cortivo L, Marolleau JP, Gluckman E, Chavinié J, Brossard Y, Benbunan M. The Paris Cord Blood Bank. Bone Marrow Transpl. 1998;22:S11.

38. Wernet PW. The International Netcord Foundation. In: Broxmeyer He, editor. Cord blood: biology, immunology, banking and clinical transplantation. Bethesda, Md: Aabb Press; 2004. P. 429-35.

39. WMDA report. Total Number of Donors and Cord blood units. https://statistics.wmda.info/; https://wmda.info/cord-blood/basicscord-blood/; 2020.

40. Mayani H, Wagner JE, Broxmeyer HE. Cord blood research, banking, and transplantation: achievements, challenges, and perspectives. Bone Marrow Transplant. 2020;55:48-61.

41. Magalon J, Gamerre M, Picard C, Chabannon C. Increase the quality of banked cord blood units without limiting HLA diversity: how cord blood banks could face this dilemma. Transfus. 2014;54:495-6.

42. Stritesky G, Wadsworth K, Duffy M, Buck K, Dehn J. Evaluation of the impact of banking umbilical cord blood units with high cell dose for ethnically diverse patients. Transfus. 2018;58:345-51.

43. Querol S, Gomez SG, Pagliuca A, Torrabadella M, Madrigal JA. Quality rather than quantity: the cord blood bank dilemma. Bone Marrow Transpl. 2010;45:970-8.

44. Feve F, Cambon-Thomsen A, Eliaou J-F, Gourraud P-A, Raffoux C, Florens J-P. Economic evaluation of the organization of a registry of haematopoietic stem cell donors. Rev Epidemiol Sante Publique. 2007;55:275-84.

45. Ruggeri A, Paviglianiti A, Gluckman E, Rocha V. Impact of HLA in cord blood transplantation outcomes. HLA. 2016;87:413-21.

46. Hough R, Danby R, Russell N, Marks D, Veys P, Shaw B, et al. Recommendations for a standard UK approach to incorporating umbilical cord blood into clinical transplantation practice: an update on cord blood unit selection, donor selection algorithms and conditioning protocols. $\mathrm{Br} \mathrm{J}$ Haematol. 2016;172:360-70.
47. Gutman JA, Ross K, Smith C, Myint H, Lee C-K, Salit R, et al. Chronic graft versus host disease burden and late transplant complications are lower following adult double cord blood versus matched unrelated donor peripheral blood transplantation. Bone Marrow Transpl. 2016;51:1588-93.

48. Lazaryan A, Weisdorf DJ, DeFor T, Brunstein CG, MacMillan ML, Bejanyan N, et al. Risk factors for acute and chronic graftversus-host disease after allogeneic hematopoietic cell transplantation with umbilical cord blood and matched sibling donors. Biol Blood Marrow Transpl. 2016;22:134-40.

49. Milano F, Gooley T, Wood B, Woolfrey A, Flowers ME, Doney $\mathrm{K}$, et al. Cord-blood transplantation in patients with minimal residual disease. N. Engl J Med. 2016;375:944-53.

50. Veys P, Danby R, Vora A, Slatter M, Wynn R, Lawson S, et al. UK experience of unrelated cord blood transplantation in paediatric patients. Br J Haematol. 2016;172:482-6.

51. Pupella S, Bianchi M, Ceccarelli A, Calteri D, Lombardini L, Giornetti A, et al. A cost analysis of public cord blood banks belonging to the Italian Cord Blood Network. Blood Transfus. Trasfus Sangue. 2018;16:313-20.

52. Sacchi N. Is it time to re-think a sustainable banking model for the Italian Cord Blood Network? Blood Transfus Trasfus Sangue. 2018;16:221-3.

53. Querol S, Rubinstein P, Marsh SGE, Goldman J, Madrigal JA. Cord blood banking: 'providing cord blood banking for a nation. Br J Haematol. 2009;147:227-35.

54. Magalon J, Maiers M, Kurtzberg J, Navarrete C, Rubinstein P, Brown $\mathrm{C}$, et al. Banking or bankrupting: strategies for sustaining the economic future of public cord blood banks. PLoS ONE. 2015;10:e143440.

55. Rubinstein P. Cord blood banking for clinical transplantation. Bone Marrow Transpl. 2009;44:635-42.

56. Allan D, Kiernan J, Gragert L, Dibdin N, Bartlett D, Campbell T, et al. Reducing ethnic disparity in access to high-quality HLAmatched cord blood units for transplantation: analysis of the Canadian Blood Services' Cord Blood Bank inventory. Transfus. 2019;59:2382-8.

57. Mugishima H, Takahashi T, Nagamura T, Asano S, Saito H. Umbilical cord blood for unrelated bone marrow replacement; Asia bank and Japan cord blood bank network update. Int J Hematol. 2002;76:284-6.

58. Kodera Y. The Japan Marrow Donor Program, the Japan Cord Blood Bank Network and the Asia Blood and Marrow Transplant Registry. Bone Marrow Transpl. 2008;42:S6.

59. Barker JN, Boughan K, Dahi PB, Devlin SM, Maloy MA, Naputo $\mathrm{K}$, et al. Racial disparities in access to HLA-matched unrelated donor transplants: a prospective 1312-patient analysis. Blood Adv. 2019;3:939-44.

60. Bochtler W, Maiers M, Oudshoorn M et al. World Marrow Donor Association guidelines for use of HLA nomenclature and its validation in the data exchange among hematopoietic stem cell donor registries and cord blood banks. Bone Marrow Transplant 2007;39: 737-41. https://doi.org/10.1038/sj.bmt.1705672.

\section{Affiliations}

Hanadi Rafii $\mathbb{D}^{1,2}$ - Federico Garnier ${ }^{3}$ - Annalisa Ruggeri $\mathbb{D}^{1,4} \cdot$ Irina lonescu $^{3}$ - Caroline Ballot ${ }^{5}$. Danièle Bensoussan ${ }^{6} \cdot$ Christian Chabannon $^{7} \cdot$ Bernard Dazey $^{8} \cdot$ John De Vos $^{9} \cdot$ Eric Gautier $^{10}$. Christine Giraud $^{11} \cdot$ Jérome Larghero ${ }^{12}$ - Audrey Cras $\mathbb{1}^{12} \cdot$ Valérie Mialou $^{13} \cdot$ Virginie Persoons $^{14}$. Fabienne Pouthier ${ }^{15}$ - Jean-Baptiste Thibert ${ }^{16}$. Jean-Hugues Dalle $\mathbb{D}^{17}{ }^{17}$. Gerard Michel $\mathbb{D}^{18}$. Chantal Kenzey ${ }^{1,2}$. Fernanda Volt ${ }^{1,2}$. Vanderson Rocha ${ }^{1,19}$ - Jacques-Olivier Bay ${ }^{20}$ - Marie-Thérèse Rubio ${ }^{21}$ - Catherine Faucher ${ }^{3}$. Evelyne Marry ${ }^{3} \cdot$ Eliane Gluckman $^{1,2}$ 
1 Eurocord, Hopital Saint-Louis, AP-HP, Institut de Recherche de Saint-Louis (IRSL) EA3518, Université de Paris, Paris, France

2 Monacord, Centre Scientifique de Monaco, Monaco, Monaco

3 Agency of Biomedecine, Saint Denis La Plaine, France

4 Haematology and Bone Marrow Transplant Unit, IRCCS San Raffaele Scientific Institute, Milan, Italy

5 Cell Therapy unit, Etablissement Français du Sang Hauts de France Normandie, site de LILLE - Belfort, Lille, France

6 Tissue Engineering and Cell Therapy unit, Regional University hospital, Nancy, France

7 Paoli-Calmettes Institute, Departement of Cancer Biology, Inserm CBT1409, Marseille, France

8 Cell Therapy unit, Etablissement Français du Sang, Bordeaux, France

9 Cell Therapy unit, University hospital, Montpellier, France

10 Cell Therapy unit, Etablissement Français du Sang, Créteil, France

11 Department of Hematology and Cell Therapy, Etablissement Français du Sang, University hospital, Poitiers, France
12 Cell Therapy Unit and Cord Blood Bank, AP-HP, Hôpital Saint Louis, Paris, France

13 Cell Therapy unit, Etablissement Français du Sang, hopital E. Herriot, Lyon, France

14 Cell Therapy and Tissue Engineering unit, Etablissement Français du Sang, Grenoble, France

15 Cell and Tissue Engineering unit, Etablissement Francais du Sang, Besançon, France

16 Cell Therapy unit, Etablissement Français du Sang, Rennes, France

17 Hopital Robert Debré, Assistance Publique - Hôpitaux de Paris, Paris, France

18 Aix-Marseille University and La Timone Children's Hospital, Marseille, France

19 Department of Hematology, Clinics Hospital, University of São Paulo Medical School, São Paulo, Brazil

20 Department of Hematology and Stem Cell Transplantation, Clermont University, Clermont-Ferrand, France

21 Department of Hematology and Stem Cell Transplantation, regional university hospital, Nancy, France 